\title{
مفهوم نظرية المعرفة
}

\section{د. آمنة عبد السلام الزائدي}

كلية الآداب- - جامعة المرقب

\section{مقدمة:}

تحتل نظرية المعرفة مكانة مرموقة بين العلوم البشرية، فهي أساس كل معرفة ونظرية

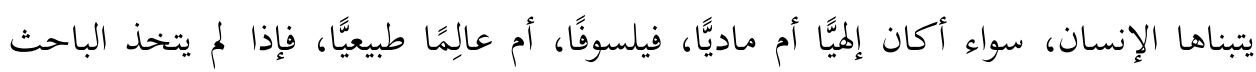

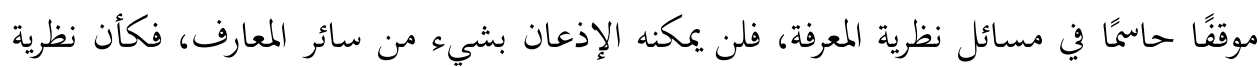

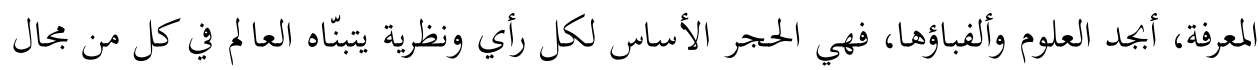
الفلسفة والعلم الطبيعي (1). فالبحث في مسائل المعرفة قديم قدم البحث في الطبيعة والإنسان، وما وراءهما، فقد كانت مسائلها بحالًا للبحث والنظر في ذلك الوقت الذي أخذ فيه بعض فلاسفة اليونان يعودون عن فلسفات أسلافهم في الوجود والعالم إلى الإنسان(2)، وإذا كان الإنسان هو مركز الدراسات الإنسانية ومحورها، فإن نظرية المعرفة هي أساس هذه الدراسات وجوهرها، من حيث تعلق دراستها، بأهم أشكال الحياة الإنسانية، وهي المعرفة البشرية. وإذا كان (( البشر جميعًا يسعون إلى المعرفة بحكم طبيعتهم )) (3) فإن هذه المعرفة هي التي تحرر العقل من الوهم، وتحرر سلوكه من الغلط، وتساعده على تمييز الحق من الباطل، 1- حسن محمد مكي العاملي: المدخل إلى العلم والفلسفة والإلميات، نظرية المعرفة، الدار الإسلامية، بيروت،

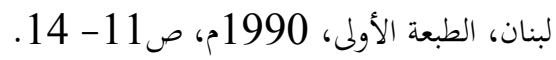
2- عبدالرممن بن زيد الزبيدي: مصادر المعرفة في الفكر الديني والفلسفي، مكتبة المؤيد، الرياض، الطبعة

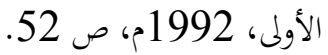

3- أرسطو: دعوة للفلسفة، ترجمة: عبدالغفار مكاوي، الهيئة المصرية العامة للكتاب، القاهرة، 1987م، 
والصدق من الكذب، والصواب من الخطإ، والطيب من الخبيث، والخير من الشر؛ ولن يتمكن

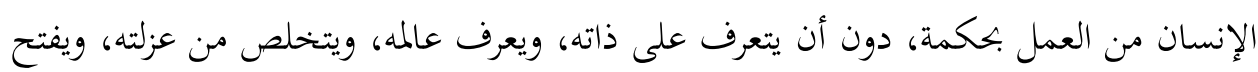

$$
\text { باب التواصل مع الآخرين عن طريق اكتساب المعرفة (1). }
$$

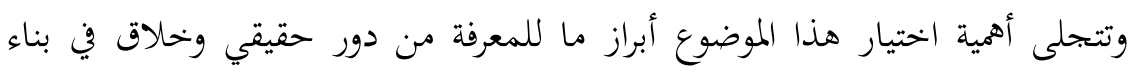

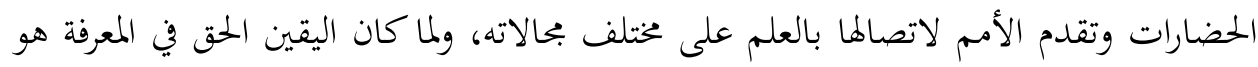
مطلب الأسمى. ولما كان هذا المطلب هو مطلب كل باحث في المعرفة؛ من هذا المنطلق اختارت الباحثة دراسة مشكلة نظرية المعرفة. لذلك حاولت الباحثة في هذه الدراسة الإجابة عن العديد من التساؤلات التي يثيرها

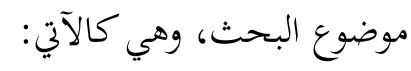
1 - ما المعرفة؟

2- 2- 2- ما الذي يمكن أن نعرفه؟

$$
\text { 3- كيف نعرف مانعرفه؟ }
$$

لذلك فإن القضية المحورية في هذا البحث تتمثل في الكشف بالبحثث والدراسة المراد

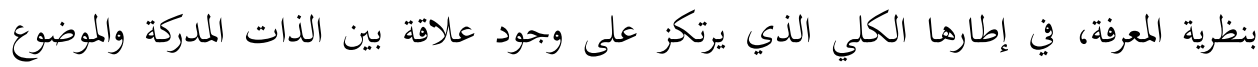

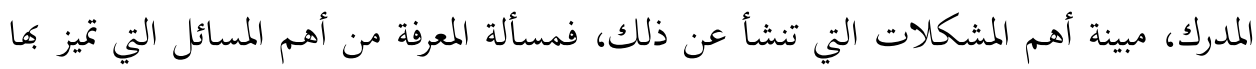

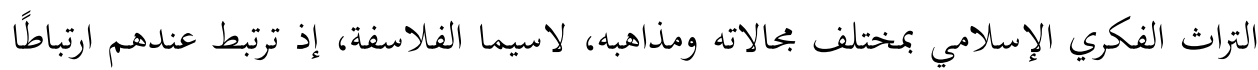

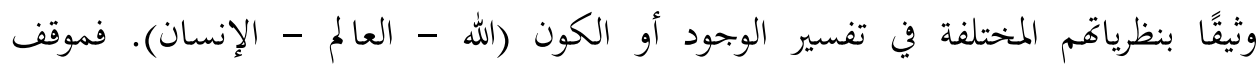

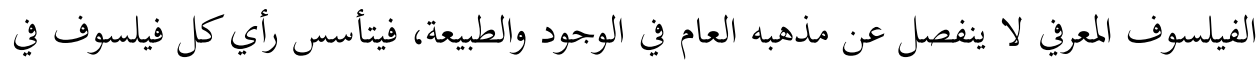
المعرفة على مذهبه في الوجود تأسسًا ذاتيًا. أما المنهج المتبع في هذا البحث فهو المنهج التاريخي والتحليلي، وقد قسم هذ البحث بعد المقدمة إلى ثلاثة مباحث، وخاتمة على النحو التالي:

1- عادل السكري، نظرية المعرفة من سماء الفلسفة إلى أرض المدرسة، الدار المصرية اللبنانية، القاهرة،

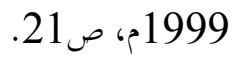




\section{مفهوم نظرية المعرفة: - مان}

وفلسفيًا تختص نظرية المعرفة بالبحث في أصل المعرفة، وطبيعتها، وحدودها، وقيمتها،

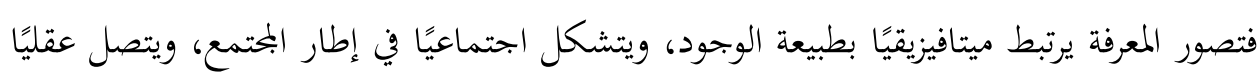
بوصف أشكال النشاط العقلي، ويرتبط منطقيًا بقواعد الاستدلال الصحيح، ويتعلق نفسيًا بعملية تكوين المعرفة، ويتحدد أخلاقيًا بالإلنزام بالحقيقة (1).

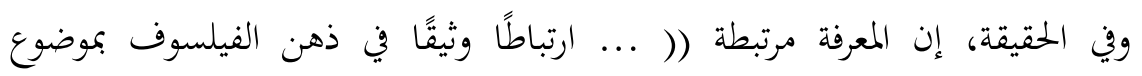
الاعتقاد، ويقصد بالاعتقاد هنا أية فكرة تسيطر على إنسان ما بحيث يهتم بها، وقد تدفعه إلى إلى

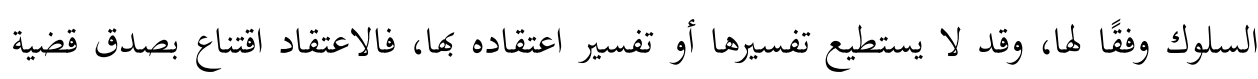
ما لأسباب مقبولة لدى المعتقد بها حتى لو لم تكن مقبولة لدى الآخرين ()(2).

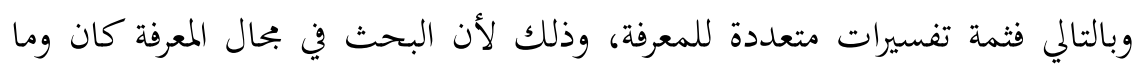

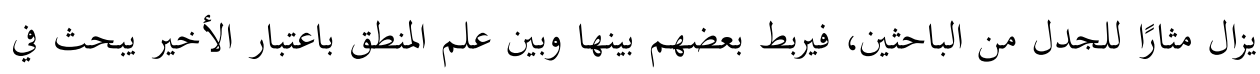

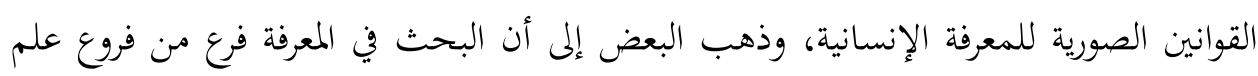

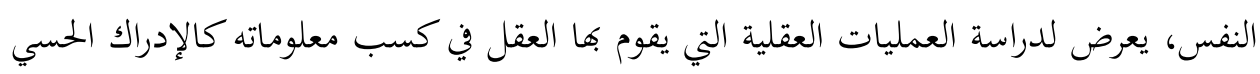

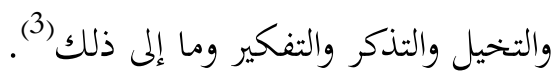
وتمة تفسير آخر لنظرية المعرفة، وهو (( أها البحث في المعرفة التي تم لنا اكتساها )) (4). كما تعرفها موسوعة الفلسفة، من حيث إها أحد فروع الفلسفة، المعني بطبيعة المعرفة وبحالها وفروضها وأصلها (5).

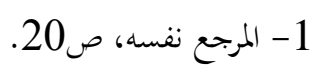

2- محمود زيدان، نظرية المعرفة عند مفكري الإسلام وفلاسفة الغرب المعاصرين، دار النهضة العربية، بيروت،

$$
\text { 1989م، ص14. }
$$

3- مصطفى النشار، المعرفة عند أرسطو، دار المعارف بمصر، الطبعة الثالثة، 1995م، صنا.

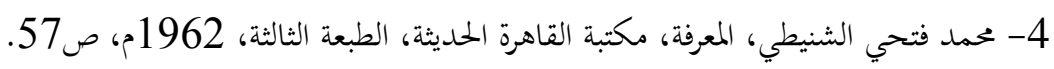

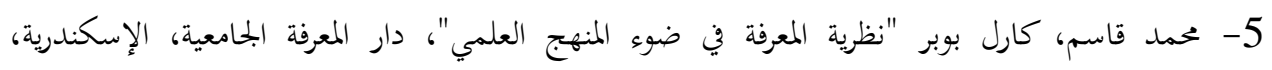
1986م، ص253. (256. 
وموضوع نظرية المعرفة في بداية الفكر الإنساني لم يكن لها وجود مستقل، ولم تفرد لها

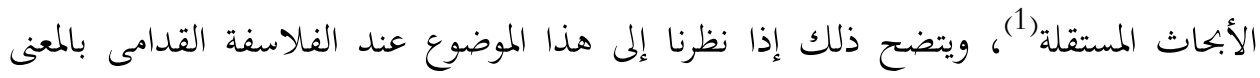

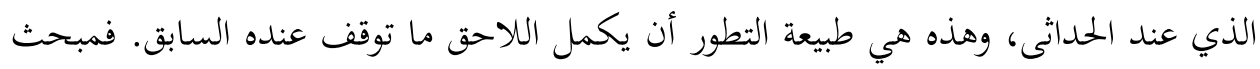

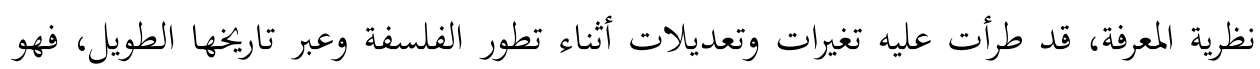

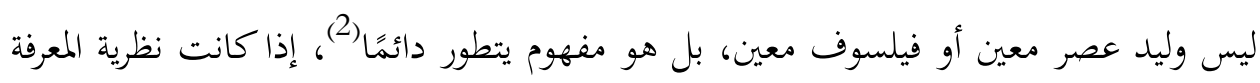

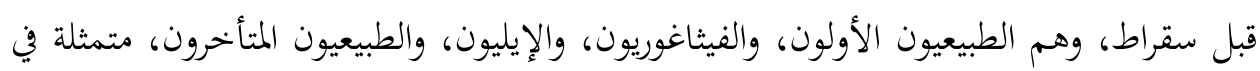

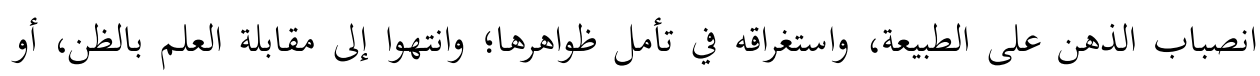

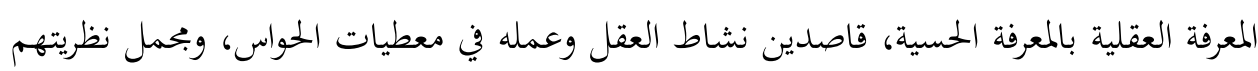

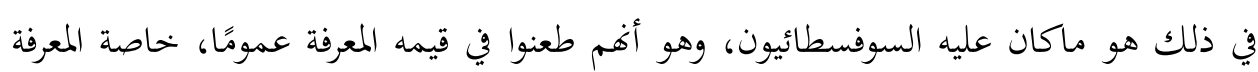

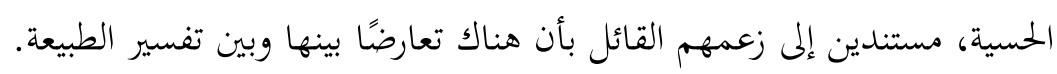

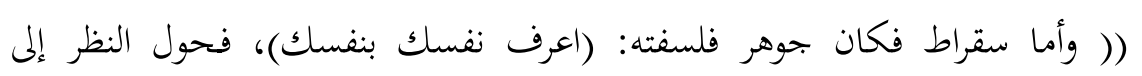

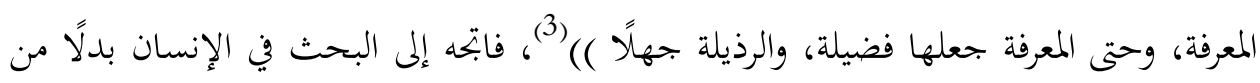

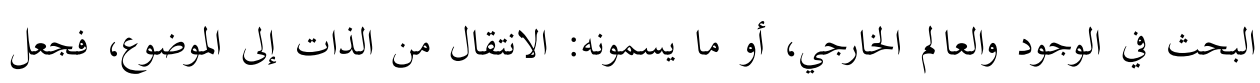
العقل مصدرًا للمعرفة، من خلال معاييره الثابتة، التي تشترك فيها العقول جميعا.

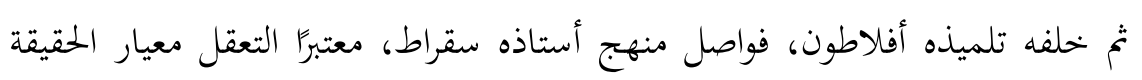

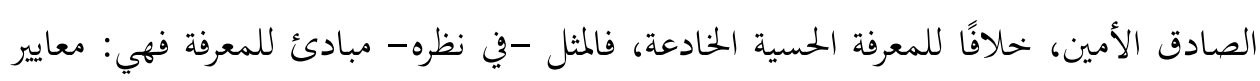

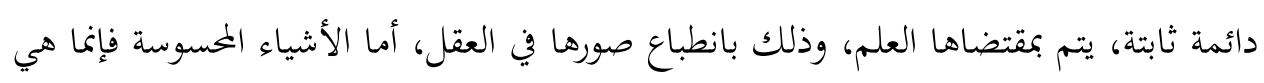
أشباح تحاكي المثل (4).

1 - م عمد فتحي الشنيطي، المعرفة، ص55.

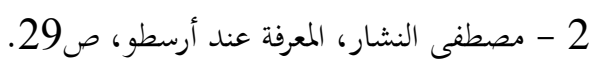

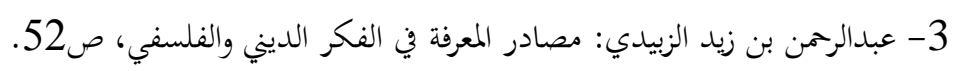

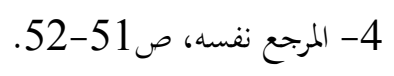


ثم جاء أرسطو من بعده وابتحه بدوره إبحاهًا مباينًا لإبحاه أفلاطون يتمثل في قوله بإننا

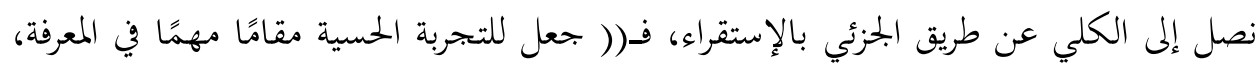
باعتبارها الأساس الذي تنهض عليه المعرفة التي يقوم بها العقل )) (1).

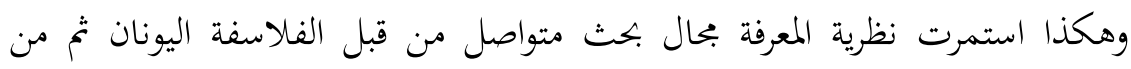
خلفهم من فلاسفة العصور الهلينستية، وتمثل هذا في مذهبي الرواقية، والآبيقورية الماديين اللذين

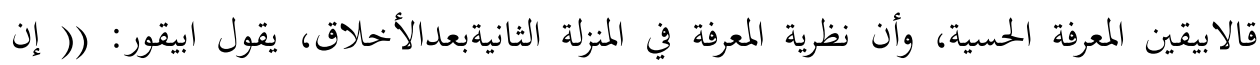

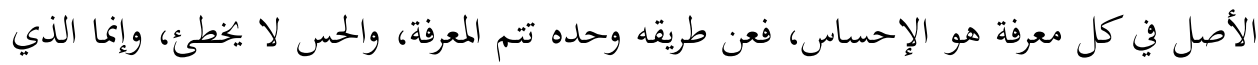
يجدث أنه تأتي إلى الحواس عن الشيء الواحد صور متعددة، والناس تختلف في التقاطها لهذه الصور، فهذا يلتقط صورة، وذلك يلتقط أخرى وهكذا . . ()) (2). والمعرفة في رأي "أبيقوراس" على أربعة أنواع هي: الإنفعال والإحساس والمعنى الكلي

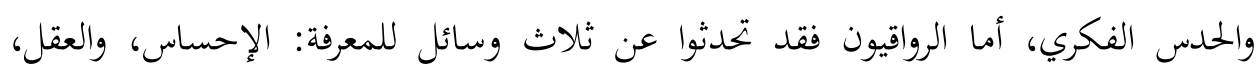
والذاكرة) والحكن

وأما عن حال نظرية المعرفة في العصور الوسطى المسيحية، فنجد أن أحد فلاسفة هذه

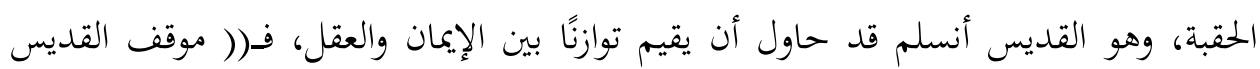
أنسلم وسط، ... إيمانية تكابر في كل مران عادي للعقل، وتصوفية تنشد حتى في الحياة الدنيا غبطة المعاينة الإلهية )) (4)، في حين أن نظرية المعرفة عند القديس توما لاكويني يمكن النظر إليها

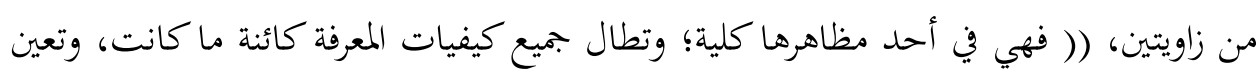

$$
1 \text { - المرجع نفسه، ص } 53
$$

2- عبدالرمن بدوي، خريف الفكر اليوناني، مكتبة النهضة المصرية، الطبعة الرابعة، القاهرة، 1970م، ص 53 3- محمد فتحي عبدالله، علاء عبدالمتعال، دراسات في الفلسفة اليونانية، دار الحضارة للطباعة والنشر،

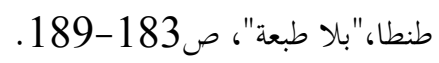
4- إميل برهييه، تاريخ الفلسفة، ترجمة: جورج طرابيشي، ج3، دار الطليعة للطباعة والنشر، بيروت، الطبعة

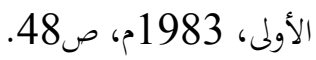


شروط كل معرفة؛ وفي مظهرها الآخر نقدية، وتعين حدود المعرفة البشرية وشروطها

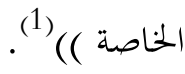

ولما جاء الإسلام بنوره، لمع من المفكرين الإسلاميين في ذلك الوقت طائفتان:

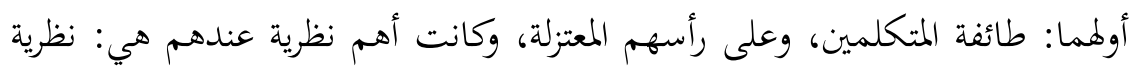

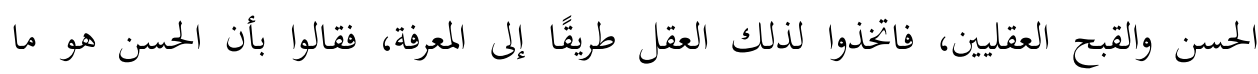
استحسنه العقل، والقبح هو ما استقبحه العقل. وكان من المتكلمين أيضًا طائفة الأشاعرة وقد سلكوا طريقًا وسطاً في المعرفة، وهي أغم جعلوا العقل في رتبة تالية للشرع، فقالوا بأن معرفة الله تحصل بالعقل وبتج بالشرع.

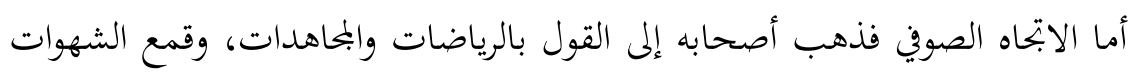

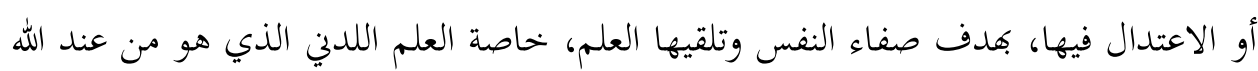
مباشرة، إضافة إلى الطريق الثاني للمعرفة وهو الحاصل بالاكتساب عن طريق الحواس.

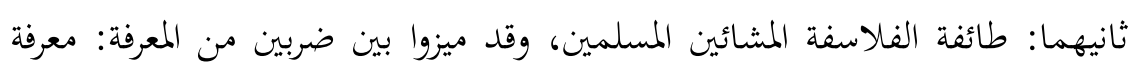

تدرك بالعقل وهي الحكمة، ومعرفة تدرك بالنص وهي الشريعة المتمثلة في القرآن والسنة. وهذا ما ركز عليه فلاسفة الإسلام في تلك الفترة، حيث أوردوا في مؤلفاقم أقوال الفلاسفة القدامى والتي جمعت بين العمليات العقلية والنفسية من حيث العقل ودوره، والنفس وقواها، والكون وموجوداته السفلية وعلاقتها بالموجودات العلوية وما فيها من العقول المفارقة، والتي منها ما أسموه بالعقل الفعال مصدر الفيض على العالم السفلي عن طريق النفس الإنسانية الناطقة والتي تعتمد في ذلك على القوة المخيلة. هذا ما بنحه عند الكندي في مؤلفاته، خصوصًا ما استعان به منها في هذا الموضوع مثل رسالته في: القول في النفس، ورسالته: في الأحلام، علاوة على تقسيمه بحالات العلوم موردًا 1- إميل برهييه، تاريخ الفلسفة، ترجمة: جورج طرابيشي، ج3،دار الطليعة للطباعة والنشر، بيروت، الطبعة

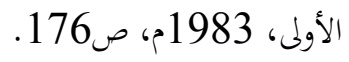


في كل ذلك أقوال أرسطو وغرضه في هذه الموضوعات ومنها غرضه في كتابه المسمى: مابعد الطبيعة.

وهذا أيضًا ما تناوله الفارابي في أكثر مؤلفاته، والتي منها كتابه الذي جمع فيه بين رأيي الحكيمين: أفلاطون وأرسطو، وكذلك رسالته: في العقل ومعانيه، وفي: المدينة الفاضلة التي

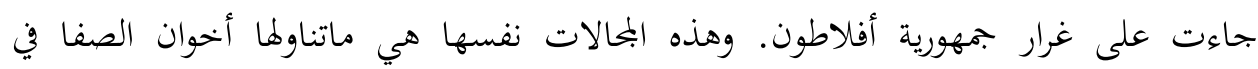

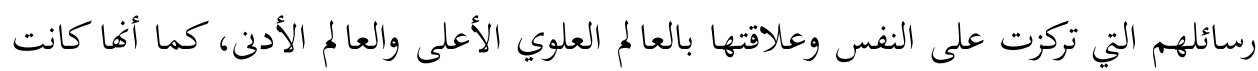
غرض ابن سينا في كتابه: الشفاء ورسائله عن: النفس وقواها وأحوالها.

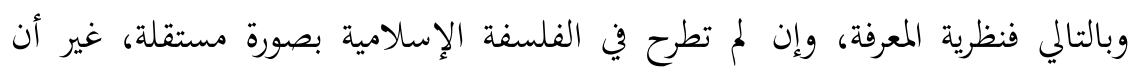

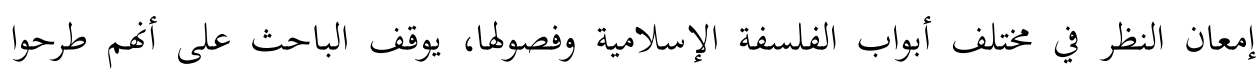
مسائلها متفرقة مبثوثة فيها، ولم ينظروا إليها كعلم مستقل (1).

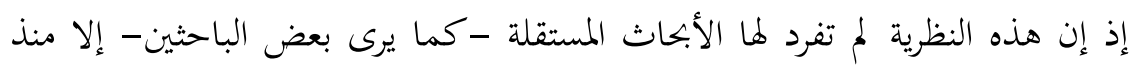

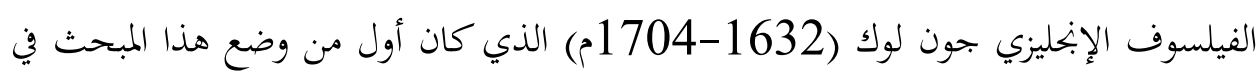

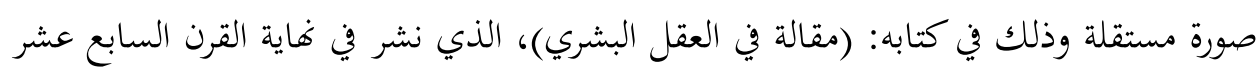

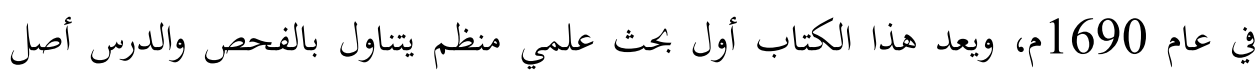

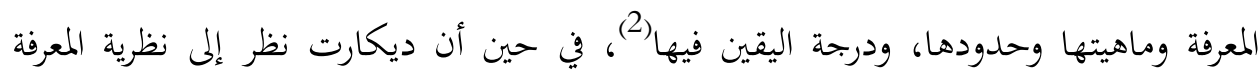

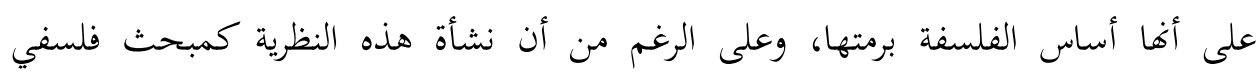

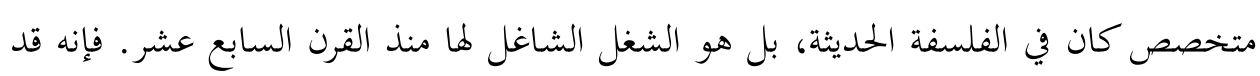
تضاءل الاهتمام بها بعد ذلك بعض الشيء (3).

1- أممد عبدالمهيمن، نظرية المعرفة بين ابن رشد وابن عربي، دار الوفاء، الإسكندرية، الطبعة الأولى، 2001م، ص.8

2- عادل السكري، نظرية المعرفة من سماء الفلسفة إلى أرض المدرسة، ص27.

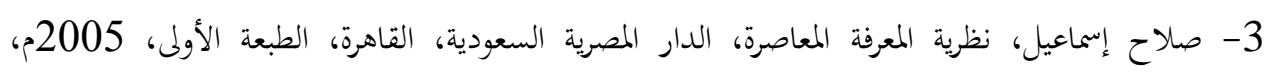


تم جاءت العقود الأخيرة من القرن العشرين لتكون شاهدة على عناية فلسفية فائقة بطبيعة المعرفة، وتحليل عناصرها الأساسية. وكذلك فـ(( الاهتمام بتحليل المعرفة لم يعد قاصرًا

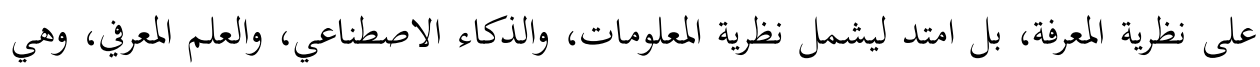
من فروع العلم التجريبي () (1). وقد ميز بعض الفلاسفة المعاصرين بين أنواع من المعرفة على سبيل المثال: المعرفة القضوية والمعرفة غير القضوية، والمعرفة القضوية: هي معرفة أن (شيئًا ما يكون كذا)، أما المعرفة غير القضوية: فتتمثل في الإدراك المباشر لشيء ما أو الإلمام به. ويرجع الفضل في التركيز على (معرفة أن) والتمييز بينها وبين (معرفة كيف) (2) إلى الفيلسوف الإنجليزي رايل (19001976م)، في كتابه المشهور (مفهوم العقل). وهناك تمييز آخر قدمه براتراندرسل (1872-1970م) وقال به أيضًا وليم جيمس (1842-1910م) وآخرون وهو التمييز بين المعرفة بالإدراك المباشر، والمعرفة بالوصف (3). لذا فليس بحافيًا للحقيقة أو مبالغًا في عرضها من يقول (( إن قضية المعرفة في الفكر الإنساني تشكل أهم مباحثه بل النقطة المعورية فيه )(4)، حتى أصبحت نظرية المعرفة اليوم في نظر الكثيرين من المفكرين مكانة الفلسفة ذاتا، بل يرى البعض أها هي الفلسفة. وبالتالي تسعى نظرية المعرفة إلى الإجابة عن جملة من الأسئلة يقع في موضع الصدارة

منها مايلي:

1- صلاح إسماعيل، نظرية المعرفة المعاصرة، الدار المصرية السعودية، القاهرة، الطبعة الأولى، 2005م، ص 21.

2- "(معرفة أن) هي معرفة أن قضية ما صادقة، و(معرفة كيف) فهي معرفة كيف ينجز المرء فعلاً ما". انظر:

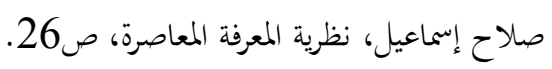

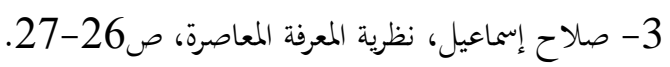

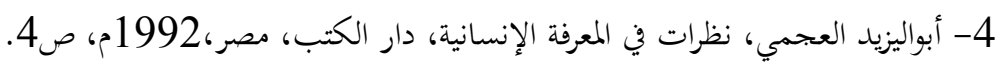




$$
\text { - } 1
$$

2- 2 - ما الذي يمكن أن نعرفه؟

$$
\text { 3- كيف نعرف ما نعرفه؟ }
$$

وهكذا يكاد مصطلح نظرية المعرفة يدل حين يطلق على البحث في: طبيعة المعرفة،

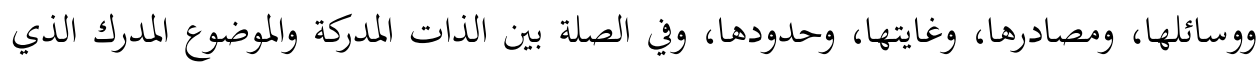

تتناوله، مما يعني أها (( .. بحموعة تنظيرات هدفها تحديد قيمة معارفنا وحدودها )ر) (1). ومن هنا فأن لفظ المعرفة حين يطلق عند المحدثين فأنه يدل على معنيين أساسيين:

الأول: الفعل العقلي الذي يتم به إدراك الظواهر الموضوعية، أي عملية الإدراك.

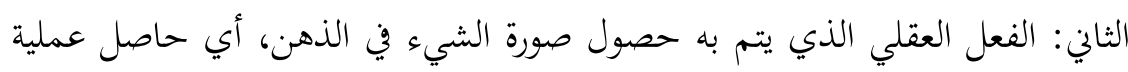

لذلك أصبح البحث في نظرية المعرفة وأن تعددت معانيها ومدلولاتما، يتضمن الإشارة

$$
\text { إلى عنصرين أساسين هما (2): }
$$

1 - وجود الذات العارفة أو أذهاننا الحاصة.

2- وجود الموضوع أو الشيء المعروف وهو ما يتضمنه العالم الخارجي.

وبناءً على ذلك فنظرية المعرفة، هي في حقيقتها مصطلح مركب من لفظين:

أولمما: نظرية، وهي تركيب عقلي مؤلف من تصورات منسقة تمدف إلى ربط النتائج بالمبادئ والمقدمات، أو هي فرض علمي يربط عدة قوانين بعضها ببعض ويردها إلى مبدأ واحد يمكن أن نستنبط منه أحكامًا وقواعد.

والثاني: المعرفة، وها تعريفات متعددة عند الفلاسفة عمومًا ثم عند المتكلمين، فهي في

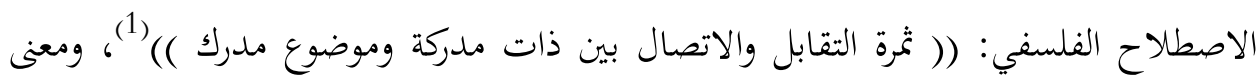

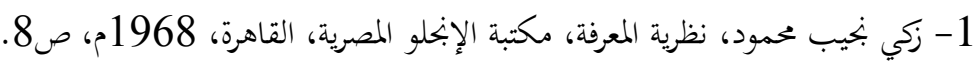

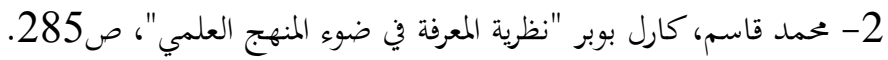


ذلك أن المعرفة عملية إدراك، فعندما يدخل الموضوع في علاقة معرفية يصبح معروفًا أي مدركًا،

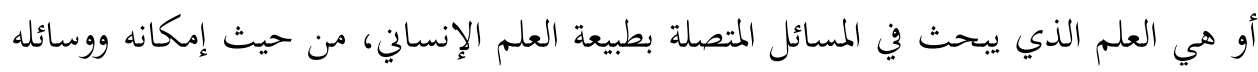

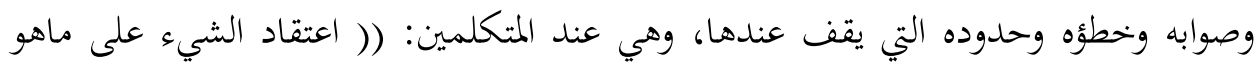
$\cdot^{(2)}(($ ب

ونستخلص -مما سبق- أن مشكلة المعرفة تتجلى في البحث في مبادىء المعرفة

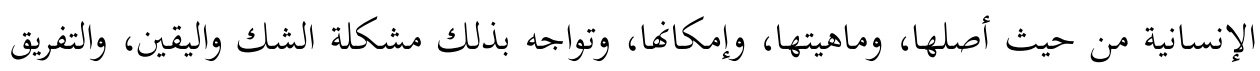

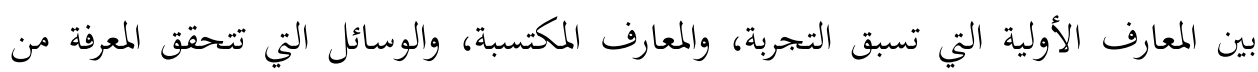

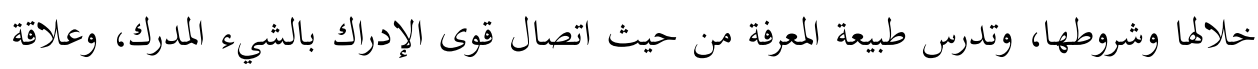

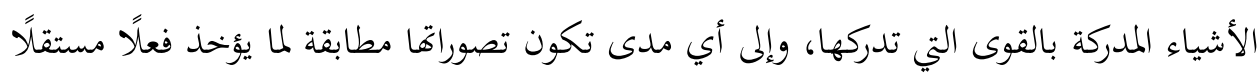

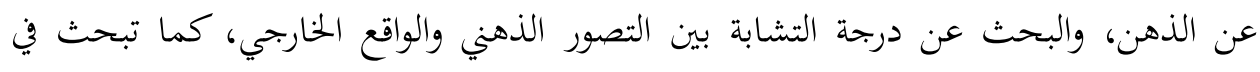

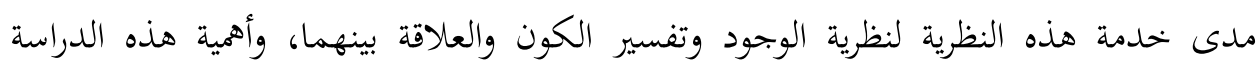

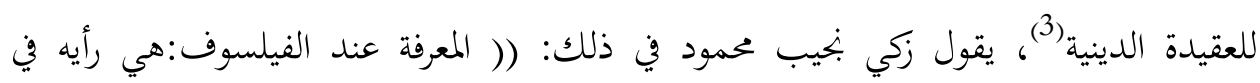

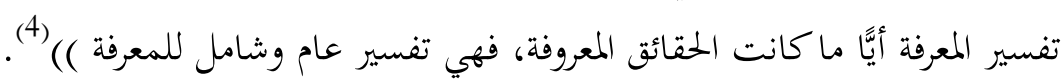

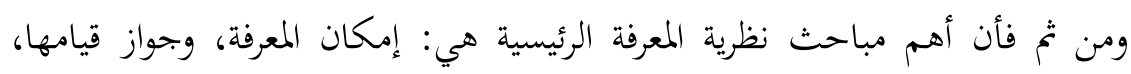

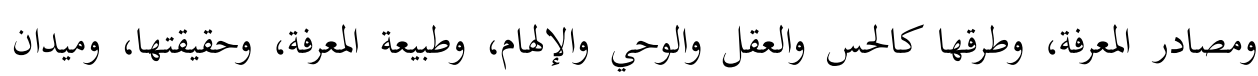
المعرفة، وحدودها، وقيمة هذه المعرفة.

1- الجرجاني، التعريفات، تحقيق: إبراهيم الأبياري، دار الكتاب العربي، بيروت، الطبعة الأولى، 1405هـ، العاري،

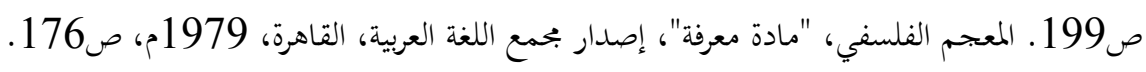

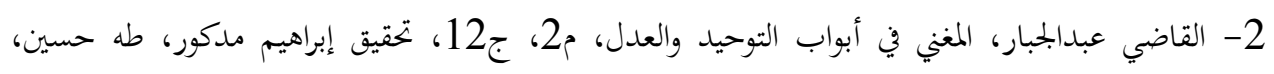

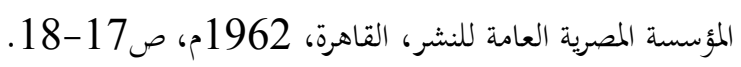
3- راجح عبدالحميد الكردي، نظرية المعرفة بين القرآن والفلسفة، مكتبة المؤيد، الرياض، الطبعة الأولى،

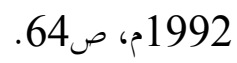

4- زكي نيب محمود، نظرية المعرفة، ص202-113، زكريا إبراهيم، دراسات في الفلسفة المعاصرة، ص28. 
ولهذا تعددت المذاهب التي تناولت نظرية المعرفة، واحتدم النزاع بينها ومن أشهرها: مذهب الشك، ومذهب اليقين، ومن ثم ظهر مذهب العقليين، ومذهب الحسيين "المذهب المثالي، والمذهب الواقعي"، وهناك المذهب الحمسي، ولكل من هذه المذاهب نظرته الخاصة إلى

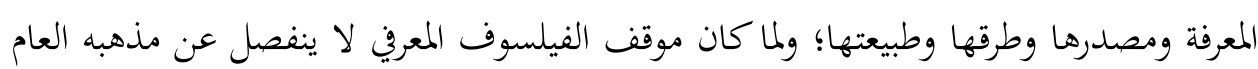
في الوجود والطبيعة، فيتأسس رأي كل فيلسوف في المعرفة على مذهبه في الوجود تأسسًا ذاتيًا،

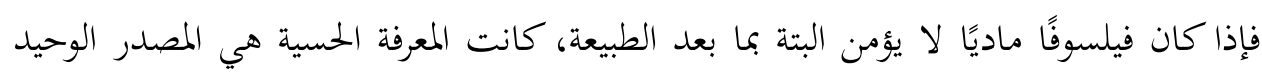

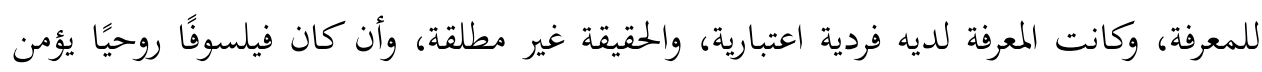
بالنفس وخلودها كانت النفس لديه أساس الملكات الإنسانية، وكانت المعرفة لديه يقينية والحقيقة مطلقة وهكذا. فهناك إذًا علاقة قوية بين موقف الفيلسوف في المعرفة وبين فلسفته في الوجود إذ أن المعرفة -كما يقال في الفلسفة- تتناسب مع الوجود، أوهي صورة منه بعبارة أدق أن.

أهم الاتجاهات والمذاهب في نظرية المعرفة: - المبة إمكان المعرفة: - إن

1- المذهب الدوجماطيقي "مذهب اليقين": القائل بإمكان المعرفة.

2- 2 مذهب الشك: المنكر لإمكان المعرفة.

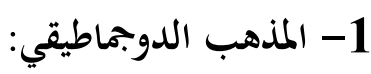

هو الابحاه الذي يؤمن إيمانًا مطلقًا بإمكان المعرفة، والقدرة على التوصل إلى اليقين، وكانت الدوجماطيقية تطلق قديمًا على كل فلسفة، تثبت حقائق معينة في مقابل مذهب الشك، الذي يمتنع عن إثبات الحقائق أو نفيها. أما حديثًا، فقد استعمل اللفظ للدلالة على التسليم بالمعرفة، دون البحث عن وجه الحق في إقرارها، في مقابل المذهب النقدي عند كانط؛ فأصحاب

1- 1 اجح الكردي، نظرية المعرفة بين القرآن والفلسفة، ص66. 
المذهب الدوجماطيقي يبدأون تفكيرهم من نقطة معينة يؤمنون بها، وينطلقون منها دون نقد أو تحليل (1)

ويككن التمييز بين نوعين من النظريات الدوجماطيقية في المعرفة:

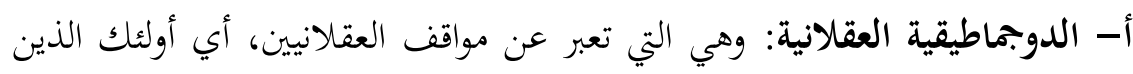

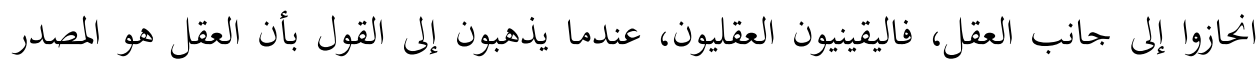

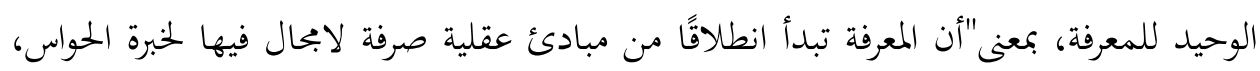
فإننا من هذه المبادئ يمكننا استنباط علم كامل بحقائق كل الأشياء" (2.).

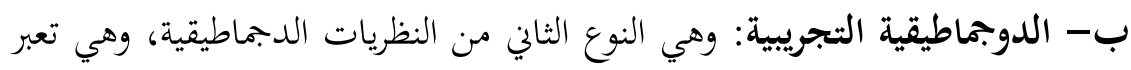

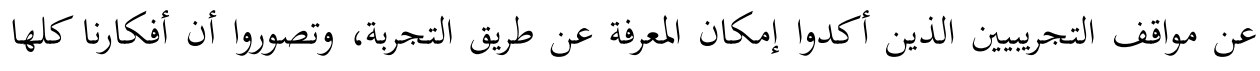

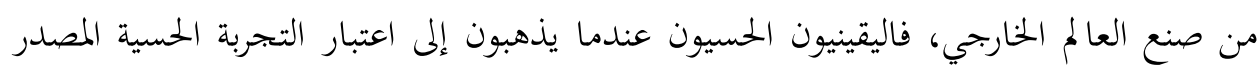

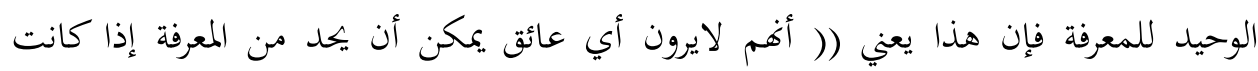

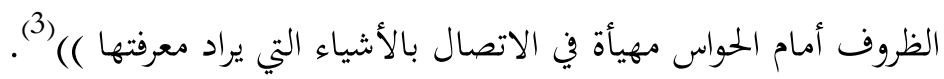

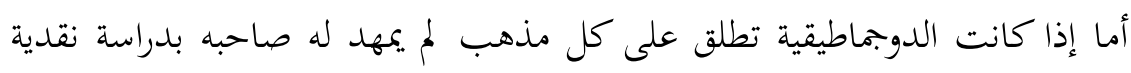
تحليلية كافية، (( فإفا بذلك لاتقتصر فقط على العقلانيين والتجريبين، بل إفا تشمل أيضا

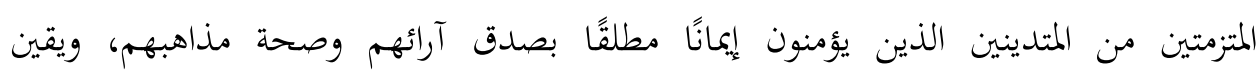

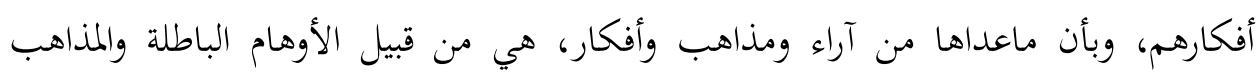

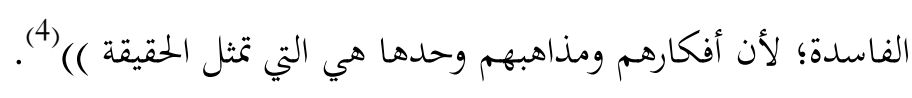

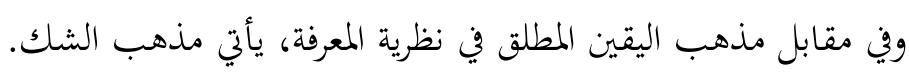

1- الكردي، نظرية المعرفة بين القرآن والفلسفة، ص33.

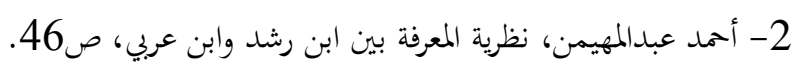

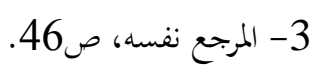

4- عادل السكري، نظرية المعرفة من سماء الفلسفة إلى أرض المدرسة، ص 36. 


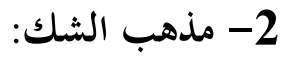

ويمكن هنا التمييز بين نوعين من الشك:

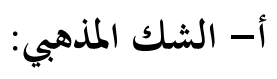

وهذا النوع الأول من الشك، وهو الشك المذهبي: (( يتخذ الإنسان من الشك منهجًا

يلغي به كل معرفة، ويبدأ به وينتهي إليه؛ فالشك هنا هو الوسيلة والغاية معًا، وهو شك

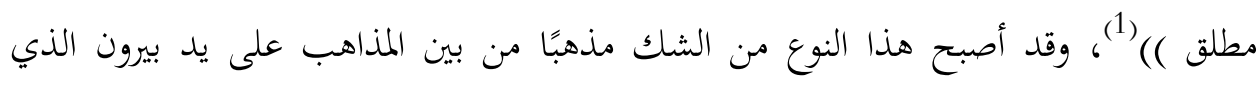
ذهب إلى (( أننا لانستطيع أن نعرف أي شيء من الأشياء، ومن ثم ... فمن الأفضل أن نتوقف عن الحكم عليها ))(2)، كما ذهب بعضهم إلى أنه لايوجد شيء وإذا وجد يستحيل معرفته، وحتى إذا عرف يستحيل نقله إلى الآخرين. فأصحاب مذهب الشك المطلق ينكرون إمكان المعرفة، بل ينكرون كل صور المعرفة، ومن ثم فالمعرفة مستحيلة. فـ(( الشكاك يرون أنه ليس للجهل الإنساني بحقائق العالم حد يقف عنده، إذ لا وسيلة أمام الإنسان في وسعه إلتماسها ليعرف شيئا خارج نفسه )(3) (3). وبالتالي فمذهب الشك المطلق هو مذهب نفاة الحقائق، حيث يزعمون أنه لا علم ولا معرفة ولا يوجد شيء له حقيقة ثابتة، فينفون كل معرفة يقينية، ويتوقفون عن إصدار أي حكم بإعتبار أن كل قضية معرفية عندهم تقبل الإيجاب والسلب (4).

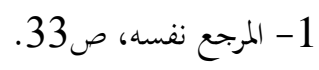

2-روزنتال بوين، الموسوعة الفلسفية، ترجمة: سمير كرم، دار الطليعة، بيروت، الطبعة الخامسة، 1985م، ص97.

$$
\text { 3- زكي بنيب محمود، نظرية المعرفة، ص105. }
$$

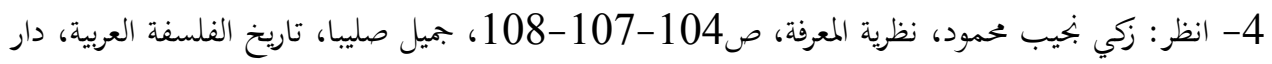

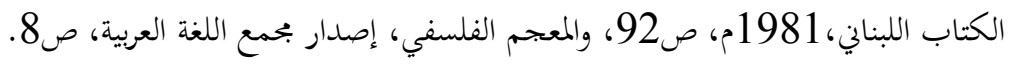




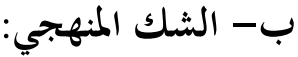

أما النوع الثاني من الشك، وهو الشك المنهجي: يعتبر المقدمة الضرورية للبحث عن

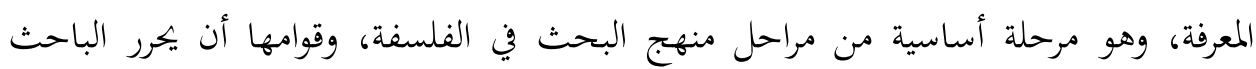

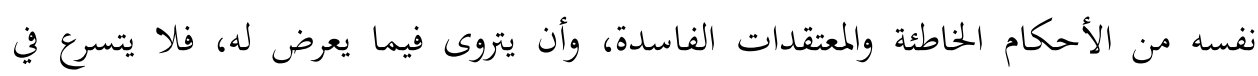
حكمة ولا يقبل إلا ما يثبت يقينه للعقل بعد الفحص والتمحيص.

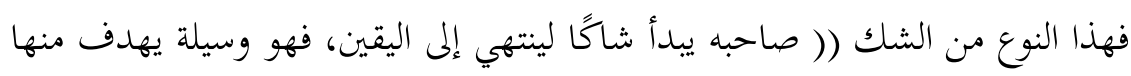

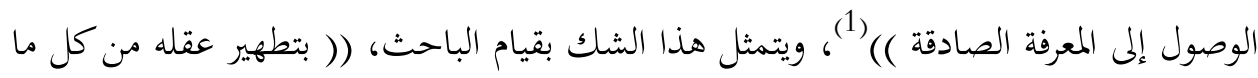

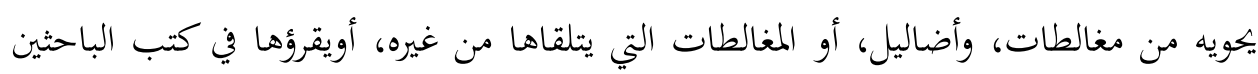

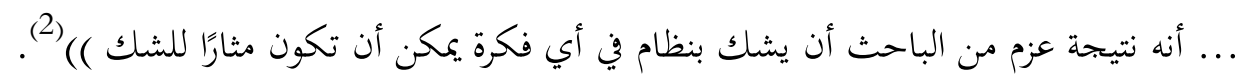

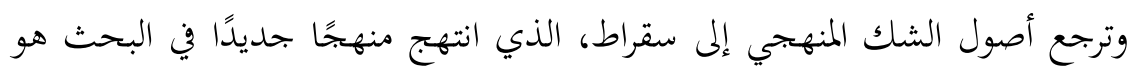
المنهج المعروف (بالتهكم والتوليد)، واستخدم أرسطو ومدرسته المشائية الشك استخدامًا منهجيًا، يتمثل في أن أرسطو كان يربط بين الشك المنهجي والمعرفة الصحيحة، ويرى ضرورة

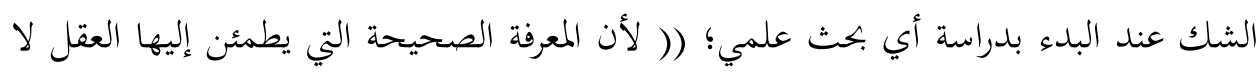

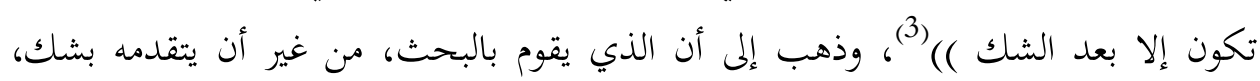

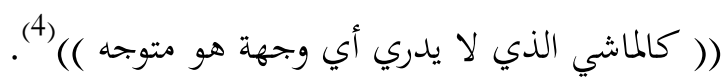
كما أكد كثير من رجال الفلسفة الحديثة على أهمية هذا النوع من الشك ولك واعتبروه ضروريًا لكل معرفة سواء في ذلك العقليون، وعلى رأسهم رينيه ديكارت، والتجريبيون من أمثال ديفيد هيوم، الذي سماه (بالشك العلمي) وقرر أن الفلسفة لابد من أن تقوم على أسس شكية.

$$
\begin{aligned}
& \text { 1- عبد الرحمن الزبيدي، مصادر المعرفة، ص63. }
\end{aligned}
$$

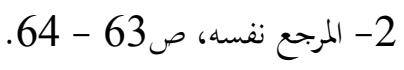

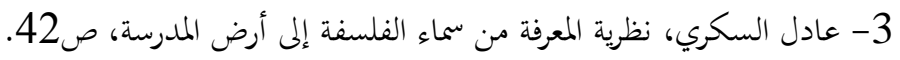

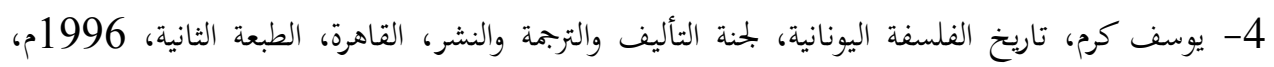




\section{مصادر المعرفة: - مصاد}

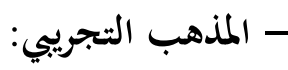

يرى التجريبيون جميعًا أن مصدر معارفنا هي الخبرة الحسية، ووسيلتنا في اكتساب المعرفة هي الحواس الخمس، ومن أكبر القائلين بهذا الاتحاه جون لوك، حيث يرى أن أي فكرة تتولد في الذهن إنما ترتد إلى مصدر واحد هو التجربة أو الخبرة الحسية، فالإنسان يولد عند جون لوك وعقله صفحة بيضاء خالية من أي معان أولية أو أي أفكار فطرية. فالإحساس سابق على لى

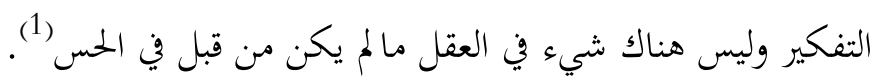
فالاتحاه الحسي إذًا هو الذي يقول أتباعه بأن جميع معارفنا ناشئة عن الاحساسات، فالتصورات القائمة في العقل الإنساني مستقاة كلها من الطبيعة. ولذلك فإن الابحاه التجريبي، قائم على أساس أن التجربة هي المصدر الوحيد للمعرفة

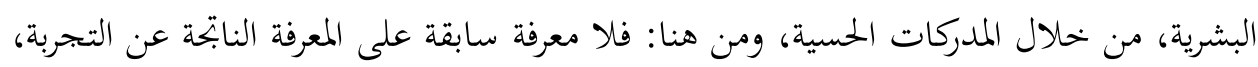
ولاطريق لمعرفة سواها(2).

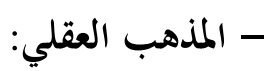

يقوم المذهب العقلي في المعرفة على أساس أن العقل هو المصدر الوحيد للمعرفة،

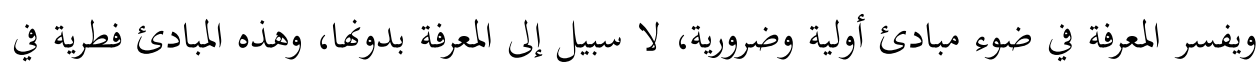

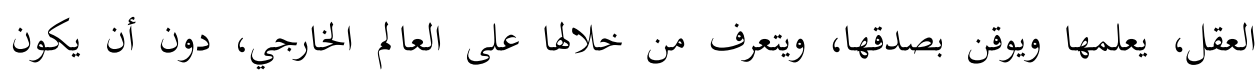
لإِحساس أو التجربة شأن فيها. ولهذا فإن مبادئه لابد من أن تكون كلية مشتركة لدى جميع الأذهان، وضرورية صادقة

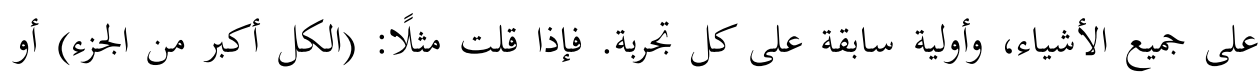
(المساويان لثالث متساويان) أو (الشيء الواحد لايمكن أن يكون موجودًا وغير موجود في وقت الته

1 - عزمي إسلام، جون لوك، دار الثقافة، القاهرة، ص44.

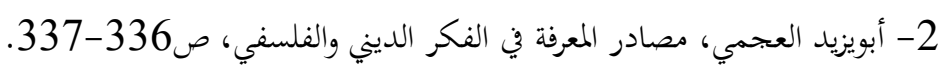


واحد)، فهذه المبادئ، وغيرها، صادرة عن العقل، (( وهي نوع من الحقائق التي لاتخطىء مرة،

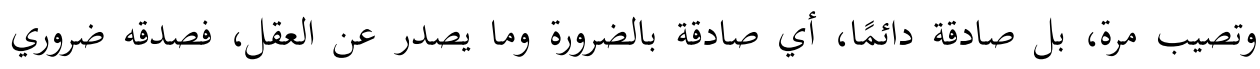
(1) () ومحتوم (1)

وهكذا يقوم موقف العقليين على أننا نستطيع عن طريق الاستدلال العقلي المخض وعلى التسليم بأن للعقل مبادئ جاهزة، وطرقًا فطرية للعمل هي التي تقوده إلى معرفة حقائق الأشياء.

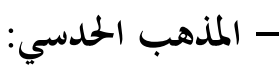

الحدس هو الإدراك المباشر للواقع أو الفهم الفوري للحقيقة، والمذهب الحدسي في نظرية المعرفة هو الذي يرد المعرفة في صورها المختلفة إلى الحدس، (( ويري أن الشعور المباشر

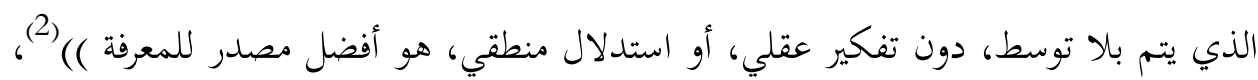
ويعتبر أن الإنسان لديه ملكة مستقلة تمكنه من فهم الحقيقة، وإدراك الواقع مباشرة، وهذه الملكة ليست حسية ولا عقلية. وبالتالي فالعقل ببراهينه وكذلك الحواس، كلاهما ليس بكافيين للوصول للمعرفة الحقة، عند أصحاب المذهب الحدسي، بل يقررون أن المصدر الحقيقي للمعرفة، إنما هو الحدس الذي يدرك الواقع بغير وساطة، ويكشف الحقيقة مباشرة، ويقودنا إلى جوهر الحياة.

\section{طبيعة المعرفة: - م}

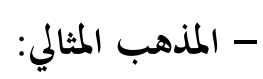

يعبر المذهب المثالي عن الاتحاه الفلسفي الذي يرد الوجود كله إلى الفكر، ويرى أن الأشياء الطبيعية لا يمكن أن يكون لها وجود بمعزل عن ذهن يعيها، وبذلك يكون جواب السؤال

$$
\text { 2- 1- زكي نجيب محمود، نظرية المعرفة، ص64 - } 65 .
$$


المطروح أمامنا ماطبيعة المعرفة؟ هو أن (( طبيعة المعرفة هي نفسها طبيعة الوجود لا فرق بين

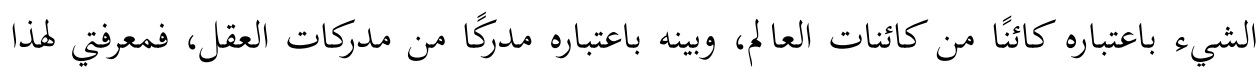

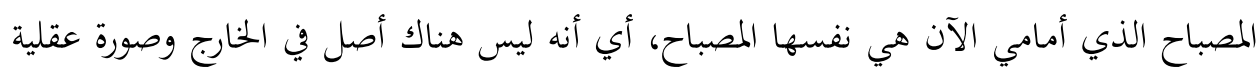

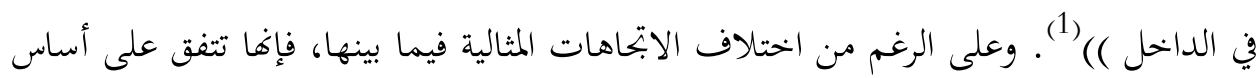

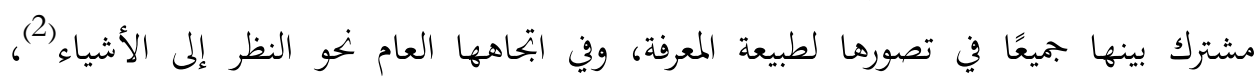

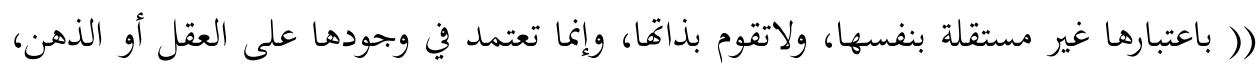

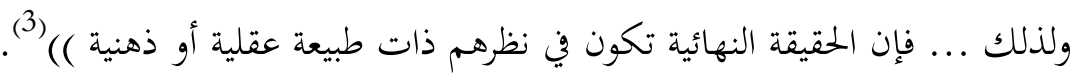

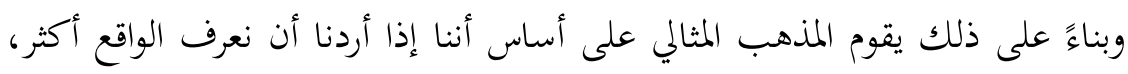

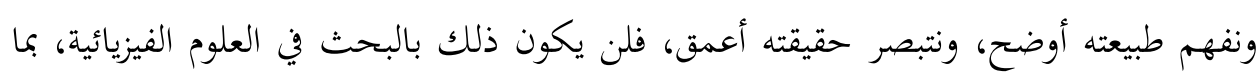

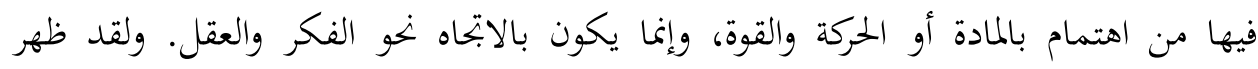

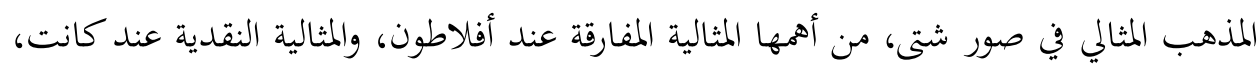
والمثالية المطلقة عند هيجل.

\section{- المذهب الواقعي:}

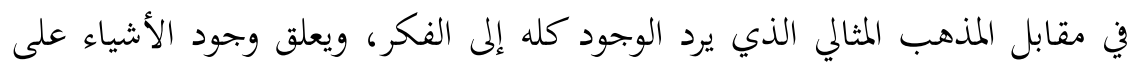

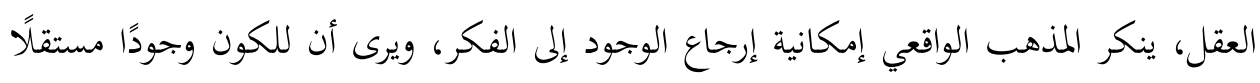

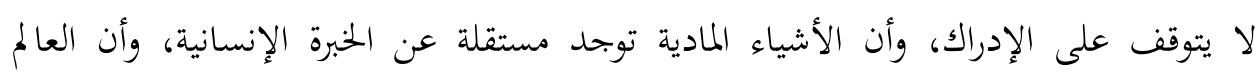
الطبيعي هو المجال الواقعي الوحيد، الذي يمكن معرفته عن طريق التجربة الحسية.

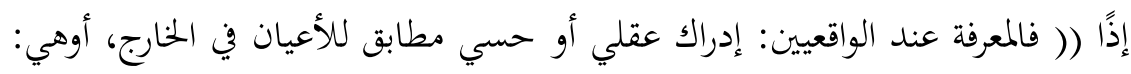

انعكاس العالم الخارجي على العقل )(|) (4).

$$
\begin{aligned}
& \text { 1- زكي نيب محمود، نظرية المعرفة، ص41. } \\
& \text { 2- }
\end{aligned}
$$

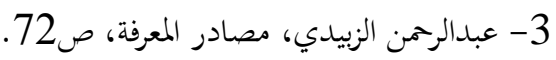

$$
\begin{aligned}
& \text { 4- المرجع نفسه، ص82. }
\end{aligned}
$$


وبالتالي فبينما ينظر المثالي إلى العالم الطبيعي باعتباره مظهرًا ماديًا للحقيقة الروحية التي

توجد وراء العالم الظاهري، والتي يتوقف عليها وجود الكون بأسره، يتجه المذهب الواقعي إلى رفض الاعتقاد بوجود عالم آخر غير العالم الطبيعي، ويرى أن الكون لايعتمد في وجوده على شيء خارج عنه، ولايكشف عن حقيقته إلا في التجربة البشرية، التي تحدث في اللحظة الحاضرة والمكان الحالي.

وقد اتخذ المذهب الواقعي أشكالًا متعددة منها:

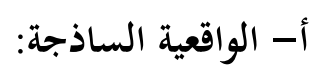

وهي الابحاه الذي ينظر إلى العالم نظرة تلقائية، ويفهمه فهمًا ماديًا ويرى الأشياء كلها توجد في العالم الخارجي مستقلة عن الوعي الإنساني، وذلك مايأخذ به عامة الناس، حين ينظرون إلى العالم نظرة مادية تلقائية، دون فحص أو نقد. فالواقعية الساذجة إذًا هي: (( الصورة الساذجة التي تثق في المدركات الحسية ثقة كاملة، وتحكم بصحة كل ماجاء عن طريقها مما أنتتت التجارب خطأه )(1).

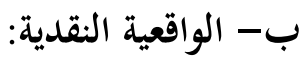

وهي الاتحاه الذي لا يتقبل العالم الخارجي كما هو، بل يخضعه للفحص والنقد، اعتمادًا على القوانين العلمية المبنية على ملاحظة العالم الموضوعي، (( فليست المعرفة تصورًا

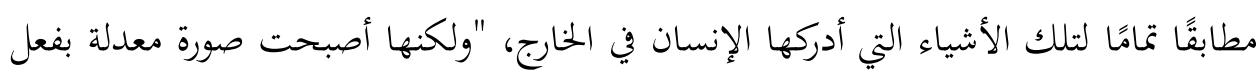

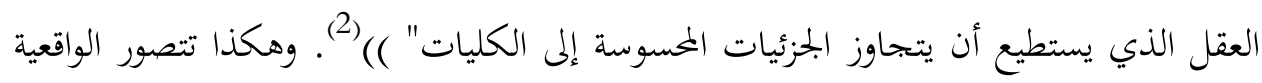

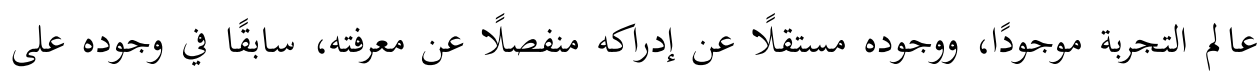
إدراك العقل له. - ماك.

$$
\text { 2- } 1 \text { - عبدالرمن الزبيدي، مصادر المعرفة، صنسه، ص83. }
$$




\section{الحخاتمة:}

- أثبت البحث أن مسألة المعرفة من أهم المسائل التي تميز بها التراث الفكري الإنساني

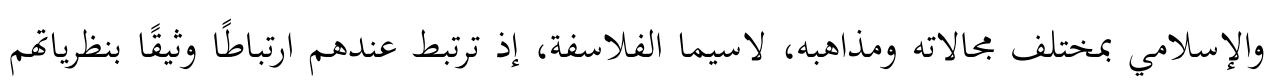
المختلفة في تفسير الوجود أو الكون (الله - العالم - الإنسان). فموقف الفيلسوف المعريف لا ينفصل عن مذهبه العام في الوجود والطبيعة، فيتأسس رأي كل فيلسوف في المعرفة على مذهبه في الوجود تأسسًا ذاتيًّا. - أثبت البحث أن مشكلة المعرفة تتجلى في البحث في مبادئ المعرفة الإنسانية، من حيث أصلها، وماهيتها، وإمكاها. فتعددت المذاهب التي تناولت نظرية المعرفة، واحتدم النزاع بينها ومن أشهرها: مذهب الشك، ومذهب اليقين، ومن ثم ظهر مذهب العقليين، ومذهب الحسيين "المذهب المثالي، والمذهب الواقعي وهناك المذهب الحدسي، ولكل من هذه المذاهب نظرته الخاصة إلى المعرفة ومصدرها وطرقها وطبيعتها. - أن الشك وسيلة لمحاربة الأوهام وأداة البحث عن الحقيقة، فلا شيء بديهيًا بذاته، لذلك لابد من اللجوء إلى الشك المنهجي أي الشك الذي يوصل إلى الحقيقة، فكان يسعى عن طريق الشك إلى ترير الفكر من الجمود والانغلاق والتحجر والتقليد. 


\section{المصادر والمراجع}

1- أبو اليزيد العجمي، نظرات في المعرفة الإنسانية، دار الكتب، مصر، 1992م، "د. ط". 2- أحمد عبدالمهيمن، نظرية المعرفة بين ابن رشد وابن عربي، دار الوفاء، الإسكندرية، الطبعة

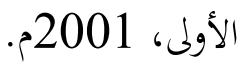
3- أرسطو: دعوة للفلسفة، ترجمة: عبدالغفار مكاوي، الهيئة المصرية العامة للكتاب، القاهرة، ." 1987 - 1987 4- الجرجاني، التعريفات، تحقيق: إبراهيم الأبياري، دار الكتاب العربي، بيروت، الطبعة الأولى، 1405 هـ 5- القاضي عبدالجبار، المغني في أبواب التوحيد والعدل، م2، ج12،تحقيق إبراهيم مدكور، طه حسين، المؤسسة المصرية العامة للنشر، القاهرة، 1962م، "د. ط". 6- إميل برهييه، تاريخ الفلسفة، ترجمة: جورج طرابيشي، ج3، دار الطليعة للطباعة والنشر، بيروت، الطبعة الأولى، 1983م. 7- جميل صليبا، تاريخ الفلسفة العربية، دار الكتاب اللبناني، 1981م، "د. ط". 8- حسن محمد مكي العاملي: المدخل إلى العلم والفلسفة والإلهيات، نظرية المعرفة، الدار الإسلامية، بيروت، لبنان، الطبعة الأولى، 1990م. 9- راجح عبدالحميد الكردي، نظرية المعرفة بين القرآن والفلسفة، مكتبة المؤيد، الرياض، الطبعة

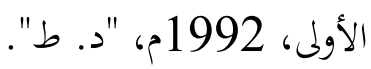


10- روزنتال بوين، الموسوعة الفلسفية، ترجمة: سمير كرم، دار الطليعة، بيروت، الطبعة الخامسة، 1985م.

11- زكي بنيب محمود، نظرية المعرفة، مكتبة الإنحلو المصرية، القاهرة، القاهرة، 1968م، "د.

12- صلاح إسماعيل، نظرية المعرفة المعاصرة، الدار المصرية السعودية، القاهرة، الطبعة الأولى، 2005

13- عادل السكري، نظرية المعرفة من سماء الفلسفة إلى أرض المدرسة، الدار المصرية اللبنانية،

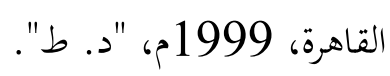

14- عبدالرحمن بدوي، خريف الفكر اليوناني، مكتبة النهضة المصرية، الطبعة الرابعة، القاهرة،

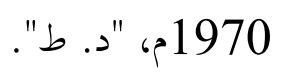

15- عبدالرحمن بن زيد الزبيدي: مصادر المعرفة في الفكر الديني والفلسفي، مكتبة المؤيد، الرياض، الطبعة الأولى، 1992م.

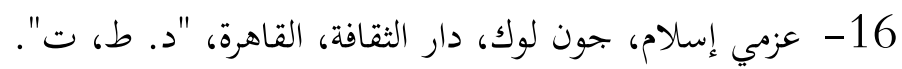
17- محمد فتحي عبدالله، علاء عبدالمتعال، دراسات في الفلسفة اليونانية، دار الحضارة للطباعة والنشر، طنطا، "د. ط". 18- محمد قاسم، كارل بوبر "نظرية المعرفة في ضوء المنهج العلمي"، دار المعرفة الجامعية، الإسكندرية، 1986م، "د. ط".

19- محمود زيدان، نظرية المعرفة عند مفكري الإسلام وفلاسفة الغرب المعاصرين، دار النهضة العربية، بيروت، 1989م، "د. ط". 
20- مصطفى النشار، المعرفة عند أرسطو، دار المعارف بمصر، الطبعة الثالثة، 1995م. 21- يوسف كرم، تاريخ الفلسفة اليونانية، لجنة التأليف والترجمة والنشر، القاهرة، الطبعة الثانية،

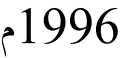

